\title{
Dimensionamento de um Sistema para Fornecimento de Energia para uma Comunidade Isolada
}

\author{
Sizing of a System for Supplying Energy to an Isolated Community \\ Danilo de Oliveira Pimentel 1,2 \\ orcid.org/0000-0003-1426-785X \\ Pedro André Carvalho Rosas 1,2 (D) orcid.org/0000-0001-9680-7228 \\ ${ }^{1}$ Escola Politécnica de Pernambuco, Universidade de Pernambuco, Recife, Brasil, \\ 2 Pós-graduação em Energias Solar e Eólica, Escola Politécnica de Pernambuco, Pernambuco, Brasil,
}

E-mail do autor principal: Danilo de Oliveira Pimentel danilopimentel87@gmail.com

\section{Resumo}

Este trabalho apresenta os resultados de um estudo prévio de viabilidade de geração de energia para uma comunidade isolada. O objetivo consiste em dimensionar e determinar um sistema de fornecimento de energia elétrica não conectado a rede de distribuição convencional para atender uma comunidade isolada específica contendo 50 famílias. A determinação desse sistema é baseado em termos de confiabilidade e custos mínimos de implantação e operação.

Utilizando o Software HOMER (Hybrid Optimization Model for Electric Renewables) e através da avaliação dos recursos solar e eólico disponível, curva de demanda da comunidade e realizando simulações com as fontes de energia disponíveis do sistema, foram obtidos os resultados que permitiram a análise de um estudo prévio de viabilidade financeira para a operação desse sistema.

Com os resultados da simulação, foram avaliados três alternativas de fornecimento de energia para a comunidade, utilizando os sistemas as fontes de energia: 1) eólica-solar-diesel-baterias, 2) solar-dieselbaterias e 3) solar-baterias, no qual o sistema solar-diesel-baterias apresentou o menor custo de energia e investimento para a comunidade.

Palavras-Chave: Sistemas Híbridos; Energia Solar; Energia Eólica; Comunidades Isoladas

\section{Abstract}

This work presents the results of a previous feasibility study of energy generation for an isolated community. The objective is to size and determine a system of electric power supply not connected to a conventional distribution network to serve a specific isolated community containing 50 families. The determination of this system is based on reliability and minimum costs of deployment and operation.

Using the HOMER Software (Hybrid Optimization Model for Electric Renewables) and by evaluating the available solar and wind resources, community demand curve and simulations with the available energy sources of the system, the results that were obtained allowed the analysis of a financial feasibility study for the operation of this system.

With the results of the simulation, three alternatives of energy supply to the community were evaluated, using the sources of energy: 1) wind-solar-diesel-batteries, 2) solar-diesel batteries and 3) solar batteries, in which the solar-diesel-battery system presented the lowest energy and investment cost to the community.

Key-words: Hybrid Systems; Solar energy; Wind Energy; Isolated Communities 


\section{Introdução}

Por muito tempo, a solução para abastecimento de comunidades isoladas se dava através do emprego de grupos de geradores a diesel, entretanto, o alto custo do óleo combustível juntamente com a logística de transporte, a manutenção do sistema de geração e a questão ambiental são fatores que tornam a aplicação desse sistema complexo e de alto custo. Contudo, as fontes renováveis de energia tais como solar (fotovoltaica) e eólica apresentam hoje possibilidades concretas para geração de eletricidades em regiões remotas, apesar de apresentar altos custos de investimento, onde uma análise criteriosa dos recursos energéticos torna-se essencial de forma a reduzir os custos [1].

Desta forma, neste trabalho foi desenvolvido uma avaliação para que obtivéssemos a melhor solução para abastecimento de energia para uma comunidade isolada contendo aproximadamente 50 famílias, de acordo com os dados de medição de velocidade de vento, dados solarimétricos e curva de carga da comunidade.

A avaliação foi executada com o suporte do software Homer Energy, que tem por objetivo simular e otimizar sistemas sistemas de geração de energia baseado em recursos renováveis. O software recebe como valores de entrada os dados solarimétricos, dados de velocidade de vento, demanda de energia e a capacidade de geração das diferentes fontes de energia do sistema, averigua a solução ótima, levando em consideração o menor custo suficiente para suprir a demanda de energia. É realizado também uma análise e decisão de como operam as fontes geradoras e o processo de carregamento e descarregamento dos bancos de bateria (sistema de armazenamento). É avaliado também os custos que envolvem o sistema, como: o valor total do investimento, valor de reposição dos equipamentos e custos relacionados a operação e manutenção.

\section{Perfil de Carga do Sistema}

A análise da demanda em função do tempo (Perfil de Carga) de um determinado grupo de consumidores (comunidade) é de extrema importância, pois ela serve de base para o dimensionamento da geração das fontes que irão atender o sistema.

O perfil de carga da comunidade em análise foi obtido através de uma previsão estimada do comportamento da comunidade ao longo do dia, onde foi levado em consideração o perfil sócio-econômico da comunidade, além disso, influências do comportamento climático ao longo do ano foram levados em conta na composição do perfil. Informações como total de unidades consumidoras atendidas (50 unidades), número total de equipamentos por unidade consumidora, conforme detalhado pela Tabela 1 , tipo, potência e regime de funcionamento dos equipamentos elétricos existentes nas unidades consumidoras e possibilidade de expansão futura do consumo de energia foram levados em consideração.

$\mathrm{Na}$ Tabela 2 é considerado o consumo médio de cargas de uso comum da comunidade.

Tabela 1 - Composição da carga por unidade consumidora considerada para levantamento do perfil carga.

\begin{tabular}{c|c|c|c|c} 
Carga & Quantidade & $\begin{array}{c}\text { Potência } \\
\text { (W) }\end{array}$ & $\begin{array}{c}\text { Utilização } \\
\text { (horas/dia) }\end{array}$ & $\begin{array}{c}\text { Consumo } \\
\text { (kwh.mês) }\end{array}$ \\
\hline Lâmpadas & 3 & 15 & 6 & 8,1 \\
\hline TV 29" & 1 & 150 & 6 & 27 \\
\hline Parabólica & 1 & 25 & 6 & 4,5 \\
\hline Liquidificador & 1 & 350 & 0,04 & 0,42 \\
\hline DVD & 1 & 10 & 0.8 & 0,24 \\
\hline $\begin{array}{c}\text { Aparelho de } \\
\text { Som }\end{array}$ & 1 & 76 & 6 & 13,68 \\
\hline $\begin{array}{c}\text { Ventilador } \\
\text { Ferro }\end{array}$ & 1 & 80 & 6 & 14,4 \\
\hline Geladeira & 1 & 90 & 24 & 64,8 \\
\hline TOTAL & & $\mathbf{1 7 9 6}$ & & $\mathbf{1 3 9 , 1 4}$ \\
\hline
\end{tabular}

A base de dados do Homer para a demanda de potência da comunidade é o conjunto de 8760 valores representando a demanda elétrica média, expressa em kW (quilowatts), para cada hora do ano. Dessa forma, foi implementado um perfil de carga diário e realizando variações nesse perfil com base nas informações das Tabelas 1 e 2 e realizando as considerações sociais, econômicas e fatores 
ambientais mencionados anteriormente obtivemos 0 perfil de demanda anual.

Tabela 2 - Consumo de cargas de uso comum da comunidade.

\begin{tabular}{c|c}
\hline Carga & $\begin{array}{c}\text { Consumo } \\
\text { (kwh.mês) }\end{array}$ \\
\hline Bombas de Água & 30 \\
\hline $\begin{array}{c}\text { Iluminação Pública } \\
\text { Outras Cargas (Escola, Capela e Centro } \\
\text { Comunitário) }\end{array}$ & 495 \\
\hline TOTAL & 750 \\
\hline
\end{tabular}

Nas Figuras 1 e 2, podemos visualizar o comportamento da demanda da comunidade para um dia típico do mês Janeiro e nas Tabelas 3 e 4 os resultados referente ao consumo diário e demanda das cargas.

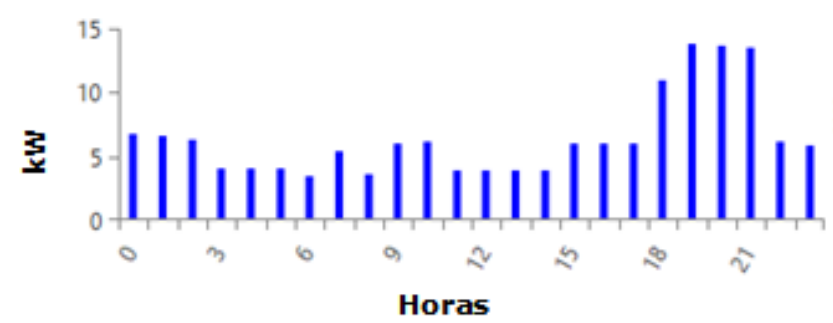

Figura 1 - Comportamento da demanda das residências ao longo do dia para um dia típico do mês de Janeiro. Fonte: Software Homer Energy Pro

Tabela 3 - Valores referentes a todas as cargas residenciais

\begin{tabular}{c|c}
\hline \multicolumn{2}{c}{ Carga Residencial } \\
\hline Consumo médio (kWh/dia) & 140 \\
\hline Demanda Média (kW) & 5.83 \\
\hline Pico de Demanda (kW) & 24.4 \\
\hline
\end{tabular}

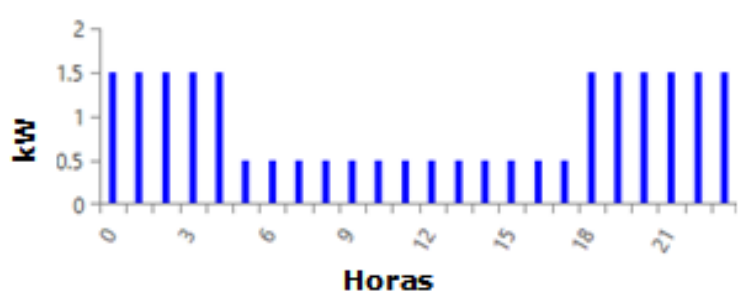

Figura 2 - Comportamento da demanda das cargas de uso comum ao longo do dia para um dia típico do mês de Janeiro.

Fonte: Software Homer Energy Pro
Tabela 4 - Valores referentes a todas as cargas de uso comum.

Fonte: Software Homer Energy Pro

\begin{tabular}{c|c}
\hline \multicolumn{2}{c}{ Cargas de Uso Comum } \\
\hline Consumo médio (kWh/dia) & 43,2 \\
\hline Demanda Média (kW) & 1.80 \\
\hline Pico de Demanda (kW) & 5.1 \\
\hline
\end{tabular}

Para o atendimento das cargas, o Homer faz uma avaliação a cada hora do ano e realiza o despacho das fontes do sistema para atender as cargas.

$\mathrm{Na}$ Figura 1, podemos notar que a demanda máxima ocorre no período entre as $18 \mathrm{~h}$ e $22 \mathrm{~h}$, ou seja, entre as primeiras horas do anoitecer, devido a ativação de cargas no sistema comumente usadas neste período como lâmpadas, televisores e ventiladores. Enquanto as menores demandas ocorrem no período da manhã, através da utilização de cargas eventuais, como exemplo o ferro de passar e liquidificador.

Outro fator que contribui para a baixa demanda registrada no período da manhã que foi levada em consideração na estimativa do perfil de carga é devido a saída dos usuários de suas residências em direção ao trabalho (atividade agrícola) e ao rio (atividade de pesca).

Na Figura 2, temos os picos de demanda, relacionados ao período de iluminação pública da comunidade compreendendo o período entre as $18 \mathrm{~h}$ de um dia típico até $05 \mathrm{~h}$ da manhã do dia seguinte. A demanda do período restante deve-se a cargas de uso comum como o funcionamento de bombas, escola e outras cargas pertencentes ao uso comunitário.

\section{Avaliação do Recurso Eólico}

Um conjunto de parâmetros são responsáveis por caracterizar o recurso eólico como: a geografia do local em estudo, dados de velocidade e direção de vento, altura da instalação de instrumentos para obtenção dos dados de vento e a variação da velocidade de vento.

Para a avaliação do recurso eólico foi considerado que a comunidade localiza-se na coordenada geográfica do local (LATITUDE - 90 $22^{\prime}$ Sul / LONGITUDE - 40²1' Oeste) como referência para a análise. Com banco de dados de 
velocidade média de vento, durante o período de um ano, obtidos de uma base de medição mais próxima da coordenada e utilizado para o processamento pelo Homer, formando assim a base de dados de velocidade média que é um conjunto de 8760 valores representando a velocidade média do vento para cada hora do ano, gerando o gráfico da Figura 3, no qual representa a velocidade de vento mensal ao longo do ano.

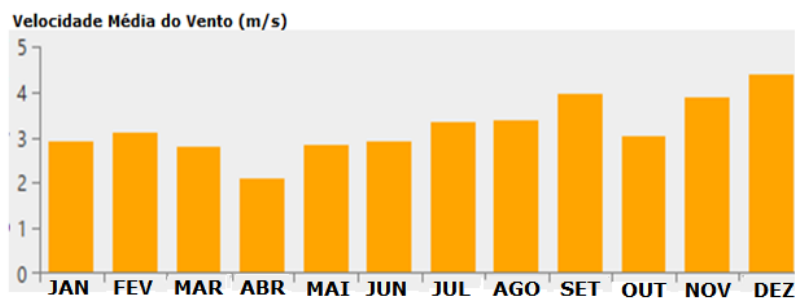

Figura 3 - Comportamento da velocidade média do vento ao longo do ano.

Fonte: Software Homer Energy Pro

A média da velocidade de vento anual registrada foi de $3.21 \mathrm{~m} / \mathrm{s}$ para uma altura de 50 $\mathrm{m}$ em relação ao nível do mar. O mês de Dezembro é o que registra a maior velocidade média de vento com $4.362 \mathrm{~m} / \mathrm{s}$ e o mês de Abril a menor com $2.094 \mathrm{~m} / \mathrm{s}$.

De acordo com o Atlas de Energia Elétrica do Brasil - ANEEL, " para que a energia eólica seja considerada tecnicamente aproveitável é necessário que sua densidade seja maior ou igual a $500 \mathrm{~W} / \mathrm{m}^{2}$, a uma altura de $50 \mathrm{~m}$, o que requer uma velocidade mínima de vento de 7 a $8 \mathrm{~m} / \mathrm{s}$ (GRUBB; MEYER, 1993) [2]". Onde, realizando uma breve comparação com as velocidades médias da base de dados, a energia eólica não apresenta ser tecnicamente viável para geração de energia para a comunidade.

\section{Avaliação do Recurso Solar}

Conhecer a radiação solar de uma localidade específica é fundamental para o projeto do sistema fotovoltaico que suprirá energia dessa área. Devido ao custo de implantação desse tipo de sistema, analisar a radiação solar é o ponto inicial para definir a utilização dessa fonte de energia.

Quando os dados solarimétricos de um determinado local são mensurados, o modo mais comum de demonstrar essas informações é em termos de energia por unidade de área (irradiação).
Utilizando a mesma coordenada da avaliação do recurso eólico, foram obtidos através da base NASA Surface Meteorology and Solar Energy Database, os dados para a análise. Esta base de dados inseridas e processadas no Homer é o conjunto de 8760 valores de radiação solar global média, expressa em $\mathrm{KWh} / \mathrm{m}^{2}$, para cada hora do ano.

Através do processamento de informações da base de dados, foi gerado o gráfico da irradiação solar global média mensal conforme a Figura 4.

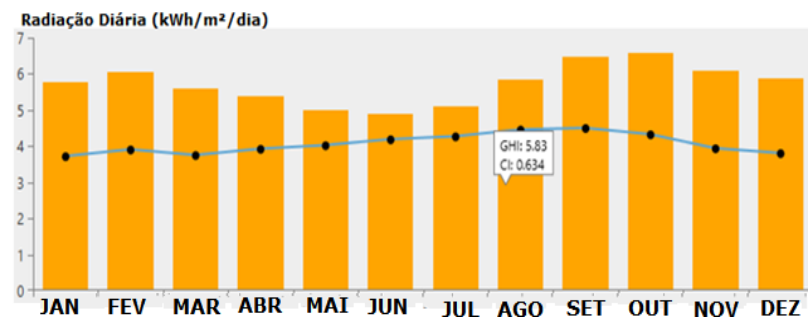

Figura 4 - Comportamento da irradiação média ao longo do ano.

Fonte: Software Homer Energy Pro

Nota-se que o mês de Outubro possui a maior média $6.580 \mathrm{KWh} / \mathrm{m}^{2} / \mathrm{d}$ de radiação e o mês de Junho a menor média com 4.880 $\mathrm{KWh} / \mathrm{m}^{2} / \mathrm{d}$. A média anual registrada de acordo com a base de dados é de $5.72 \mathrm{KWh} / \mathrm{m}^{2} / \mathrm{d}$.

Conforme o Atlas Brasileiro da Energia Solar [3], a região Nordeste do Brasil, no qual se localiza a comunidade, apresenta índices elevados de irradiação solar direta e são o principal requisito de viabilidade para a geração fotovoltaica, onde o estudo realizado para a região Nordeste apresenta um valor médio do total diário da irradiação global horizontal de 5,49 kWh/m².

\section{Geração a Diesel}

Os geradores a Diesel por muito tempo foram utilizados como única fonte de geração para comunidades ou áreas isoladas, porém o alto custo operacional e as emissões de poluentes tornam ineficaz a utilização somente dessa fonte.

Entretanto, as fontes renováveis de energia como a solar e eólica vêm se tornando bastante viáveis em termos econômicos e ambiental nos últimos anos. Consequentemente, após a avaliação dos recursos renováveis de uma região, nota-se que é possível a combinação de fontes de energia para formar um sistema, que recebe a denominação de sistema híbrido. 
Conforme o manual de instruções da EPE [4], "Por definição, sistema híbrido é um sistema gerador de energia elétrica que utiliza mais de uma fonte de energia e, ao integrá-las, é possível superar as limitações inerentes a cada uma delas. Além disso, tais sistemas têm por objetivo gerar energia elétrica de forma otimizada, dependendo da disponibilidade dos recursos, e com custos mínimos."

Por isso, é bastante usual e importante nos projetos de implantação de sistemas híbridos a utilização de geradores a diesel combinado com fontes renováveis como a eólica e solar, pois eles permitem o aumento da confiabilidade do sistema, atuando em momentos de indisponibilidade do sistema renovável ou escassez das fontes solar e eólica, o sistema de geração a diesel entra para garantir a continuidade do fornecimento de energia elétrica aos consumidores.

\section{Sistema de Armazenamento}

Conforme podemos observar anteriormente nos Tópicos 3 e 4, os recursos eólicos e solares são variáveis do tempo, mas também podem sofrer variações imprevisíveis da atmosfera. Desta forma, em diversas situações durante o ano vão existir momentos que a potência fornecida pelas fontes eólica e solar serão maiores ou menores do que a demandada pela carga.

Portanto, em sistemas híbridos para geração de energia é bastante comum o uso de sistemas de armazenamento, para que no caso de uma geração de energia excedente a demanda da carga, realizarmos o processo de armazenamento. Do mesmo modo para que em situações em que a geração for menor do que a demanda possamos utilizar essa energia.

\section{Inversor}

O inversor é um equipamento de expressiva importância dentro do sistema híbrido, visto que ele é um dos componentes responsável pelo abastecimento de energia. Ele realiza a conversão de potência (caso o sistema necessite de tal operação) de CC-CA, proveniente da geração de energia solar ou da energia armazenada nas baterias.

\section{Avaliação das Possibilidades de Abastecimento de Energia}

Após a análise da demanda (consumo a ser atendido) do sistema e do recurso disponível das fontes solar e eólica na área da comunidade, é necessário definir as fontes de energia que irão fazer parte do sistema.

Os sistemas híbridos tem uma grande vantagem de poder realizar o aproveitamento de mais de uma fonte de geração de energia e em determinados momentos realizar a complementaridade entre essas fontes.

Para o processo de simulação, foi definido inicialmente um sistema híbrido de referência, contendo três fontes (geração diesel, eólica e solar), no qual foi definido uma maior participação da fonte solar, visto que na avaliação dos recursos, a fonte eólica apresentou velocidades médias baixas para geração de energia e a fonte solar bons índices de irradiância.

Para o dimensionamento do sistema híbrido e estimativa de produção de energia através do Homer, os dados de entrada como os recursos eólicos e solares, potência ( $\mathrm{kW}$ ) de cada fonte do sistema, capital inicial de investimento, custo de reposição e custo de operação e manutenção foram definidos.

Inicialmente, foi definido para as fontes:

\section{- Solar}

Conforme as Tabelas 3 e 4, a demanda de pico máxima do sistema é de $29.5 \mathrm{~kW}$. Logo, definimos uma capacidade instalada mínima de 30 kW para a fonte solar.

\section{- Eólica}

Com base nos dados de recursos eólicos apresentados, foi selecionado para a simulação o aerogerador BWC Excel-S com potência de $10 \mathrm{~kW}$ no banco de dados do Homer, no qual é capaz de gerar energia a velocidade de vento igual ou superior $4 \mathrm{~m} / \mathrm{s}$, o comportamento do aerogerador (Figura 6) é registrado na sua curva de potência.

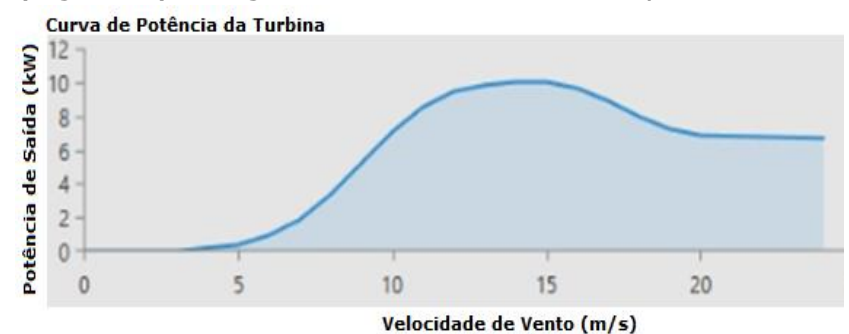

Figura 5 - Curva de potência do aerogerador Fonte: Software Homer Energy Pro 
- Gerador Diesel

A demanda média do sistema é de 7.63 $\mathrm{kW}$, logo para simulação foi definido um gerador a diesel com potência de $10 \mathrm{~kW}$, com características operacionais que podem ser observadas na curva de consumo e eficiência registrado na Figura 7.

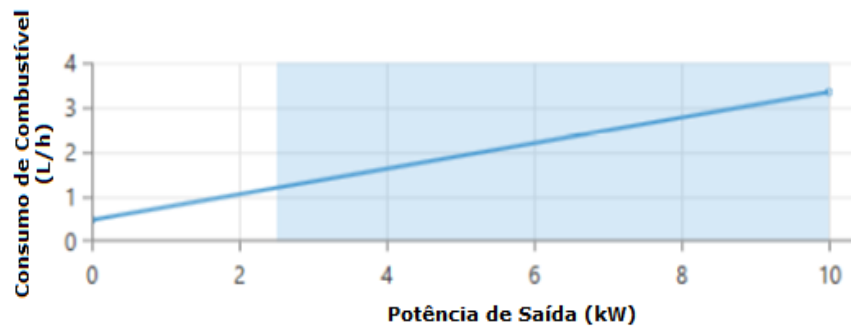

Figura 6 - Curva de consumo de combustível x potência do gerador a diesel

Fonte: Software Homer Energy Pro

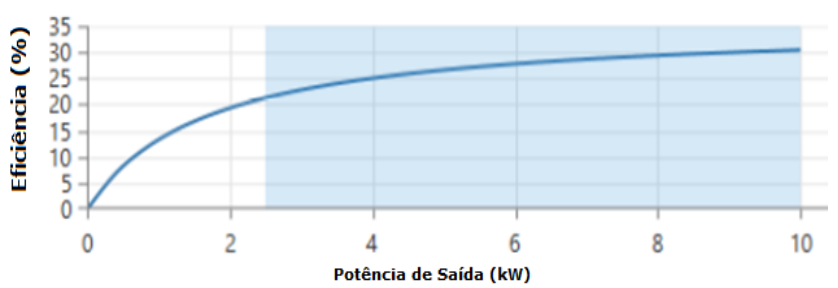

Figura 7 - Curva de eficiência do gerador a diesel Fonte: Software Homer Energy Pro

- Baterias

Inicialmente, o número mínimo de baterias para a simulação do sistema foi definido em função do consumo total diário da comunidade, registrado em torno de $183 \mathrm{kWh} / \mathrm{d}$. No banco de dados do Homer, a bateria com as seguintes características operacionais foi selecionada:

- Capacidade Nominal - 7.55 kWh

- Capacidade Máxima - 1890 Ah

- Vida Útil - 12 a 20 anos

De posse desses dados, foi estabelecido um mínimo de $\mathrm{n}=\frac{\text { Consumo diário }}{\text { Capacidade Nominal da Bateria }}=25$ baterias, para a simulação.

- Inversor

O inversor no sistema isolado deve ser definido de tal forma que possa atender a demanda máxima instalada, conforme visto anteriormente esse valor é de $29.5 \mathrm{~kW}$, foi selecionado para a simulação um inversor com potência de $40 \mathrm{~kW}$.

A estratégia utilizada é realizar uma análise que permita definir um sistema de geração que garanta confiabilidade e que minimize os custos de implantação e operação. Portanto, o critério utilizado para a definição do sistema híbrido será o que apresentar o menor custo da energia elétrica gerada ( $R \$ / k W h)$.

De acordo com os resultados das simulações, foi realizado a análise de três configurações (sistemas) que apresentaram os menores custos de energia e de operação, que são os sistemas:

1) Solar-Eólico-Diesel-Baterias

2) Solar-Diesel-Baterias

3) Solar - Baterias

\section{1 - Sistema Solar - Eólico - Diesel - Baterias}

configuração é:

O resultado da simulação para a

- Gerador Diesel - 1 x 10kW

- Gerador Fotovoltaico - 69,9 kW

- Gerador Eólico - 1 x 10 kW

- Baterias - 67 x $1890 \mathrm{Ah}$ (7.55 kWh)

- Inversor - $20 \mathrm{~kW}$

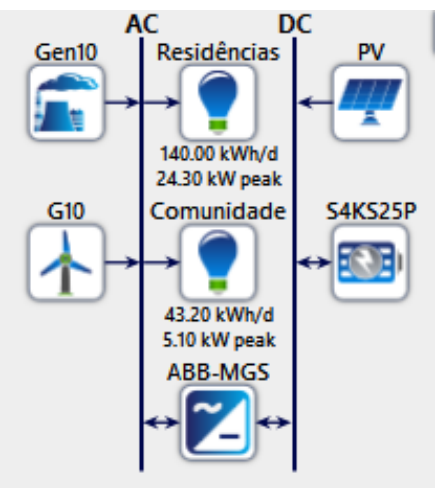

Figura 8 - Sistema Híbrido Solar - Eólico - Diesel Baterias

Fonte: Software Homer Energy Pro

Os resultados apresentados na simulação desse sistema híbrido são destacados na Tabela 5 e Figura 9:

Tabela 5 - Produção e Consumo anual de energia do sistema

Fonte: Software Homer Energy Pro

\begin{tabular}{c|c|c}
\hline Fonte & $\begin{array}{c}\text { Energia } \\
\mathbf{( k W h / a n o )}\end{array}$ & $\begin{array}{c}\text { Percentual } \\
(\mathbf{\%})\end{array}$ \\
\hline Geração Fotovoltaica & $124.830,0$ & 97.1 \\
\hline Geração Eólica & $1.963,0$ & 1.62 \\
\hline Geração a Diesel & $1.763,0$ & 1.40 \\
\hline Total & $128.555,0$ & 100 \\
\hline Consumo & $66.837,0$ & -
\end{tabular}



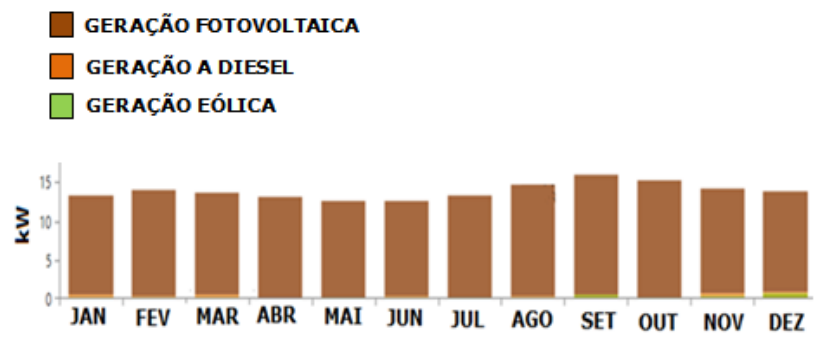

Figura 9 - Produção mensal de energia do sistema Fonte: Software Homer Energy Pro

Na Figura 10, é apresentado o gráfico que demonstra o comportamento do sistema em relação ao fornecimento de energia e a demanda solicitada pelo sistema para os primeiros dias do mês de Abril. A demanda (cor vermelha) do sistema é suprida pela fonte solar (laranja) e pelas baterias (cor verde). Podemos notar que não há registros de produção de energia eólica durante esse período e que o gerador a diesel (cor preta) é acionado em períodos de indisponibilidade da fonte solar, funcionando somente em momentos para complementar a energia fornecida pelas baterias.

A vantagem desse sistema é que ele reduz o consumo de óleo diesel, por apresentar duas fontes renováveis de energia.

GERAÇÃO FOTOVOLTAICA

GERAÇÃO A DIESEL

DEMANDA DO SISTEMA

SISTEMA DE ARMAZENAMENTO
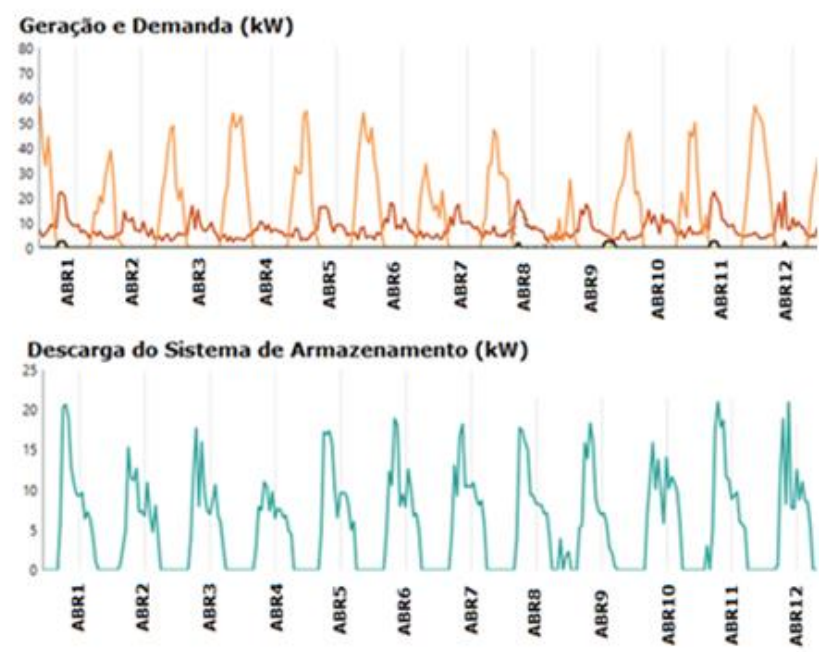

Figura 10 - Comportamento do sistema para os primeiros dias do mês de Abril

Fonte: Software Homer Energy Pro

\subsection{1 - Avaliação Financeira}

De acordo com a Tabela 6, temos uma avaliação dos custos estimados dos principais componentes do sistema.

Tabela 6 - Custos estimados associados ao sistema SolarEólico-Diesel-Baterias

\begin{tabular}{|c|c|c|c|}
\hline Componente & \begin{tabular}{l}
\multicolumn{1}{c}{ Custo de } \\
Investimento \\
inicial $(\mathbf{R} \$)$
\end{tabular} & $\begin{array}{c}\text { Custo de } \\
\text { O\&M } \\
\text { (R\$/ano) }\end{array}$ & $\begin{array}{l}\text { Custo de } \\
\text { Reposição } \\
\text { (R\$/ano) }\end{array}$ \\
\hline Subsistema Solar & $370.467,05$ & $3.704,67$ & $-*$ \\
\hline $\begin{array}{l}\text { Subsistema } \\
\text { Eólico }\end{array}$ & $150.000,00$ & $3.000,00$ & $2.507,31$ \\
\hline $\begin{array}{l}\text { Subsistema de } \\
\text { Armazenamento }\end{array}$ & $372.000,00$ & $7.440,00$ & $11.962,79$ \\
\hline Gerador a Diesel & $25.000,00$ & $9.186,71$ & $-*$ \\
\hline Inversor & $35.000,00$ & 750,00 & 933,84 \\
\hline $\begin{array}{l}\text { Controle e } \\
\text { Supervisão }\end{array}$ & $40.000,00$ & $2.000,00$ & - \\
\hline Acessórios & $20.000,00$ & $-*$ & $-*$ \\
\hline $\begin{array}{l}\text { Rede de } \\
\text { Distribuição }\end{array}$ & $45.000,00$ & $2.500,00$ & -* \\
\hline Total & $1.012 .467,05$ & $28.581,38$ & $15.403,94$ \\
\hline
\end{tabular}

-* Não previstos custos de O\&M e reposição para os itens em destaque.

Definindo o horizonte de planejamento financeiro do sistema em $\mathrm{n}=25$ anos $\mathrm{e}$ considerando uma taxa mínima de atratividade $\mathrm{TMA}=12 \%$, levamos em conta nessa análise apenas as despesas do sistema, obtivemos então através dos indicadores financeiros definidos nas equações (1), (2) e (3), os resultados da Tabela 7.

VPa - Valor presente associado a uma anuidade, onde "Va" representa o valor anualizado do custo.

$$
V p a=V a \frac{(1+T M A)^{n}-1}{i(1+T M A)^{n}}
$$

VPL - Valor Presente Líquido, onde Fcn representa o fluxo de caixa no período.

$$
V P L=\sum_{n=0}^{N} \frac{F c n}{(1+T M A)^{n}}
$$

VPLA - Valor Presente Líquido Anualizado

$$
V P L A=V P L \frac{T M A(T M A+1)^{n}}{(1+T M A)^{n}-1}
$$

Tabela 7 - Indicadores de custos do sistema

DOI: $10.25286 /$ repa.v5i3.1265 
Fonte: Software Homer Energy Pro

\begin{tabular}{l|c}
\multicolumn{1}{c|}{ Indicador Financeiro } & Valor (R\$) \\
\hline Custo de Investimento Inicial (R\$) & $1.021 .329,86$ \\
\hline Custo de O\&M - Vpa (R\$) & $224.452,78$ \\
\hline Custo de Reposição - Vpa (R\$) & $120.815,25$ \\
\hline Custo total (VPL) (R\$) & $1.357 .450,07$ \\
\hline Custo total anualizado (VPLA) (R\$) & $173.074,88$ \\
\hline
\end{tabular}

Portanto, podemos definir o custo da energia para este sistema, de acordo com a equação (4):

$$
\text { Custo da Energia }=\frac{\text { Vpla }}{\text { Energia Consumida }(\text { Anual })}
$$

Como de acordo com a Tabela 5, a energia consumida anual é igual a $66.837,0 \mathrm{kWh} /$ ano e o VPLA $=R \$ 173.074,88$, logo o custo da energia é de $\mathrm{R} \$ 2,59 / \mathrm{kWh}$.

\section{2 - Sistema Solar - Diesel - Baterias}

Nessa segunda configuração conforme a Figura 11, a simulação do sistema sem a fonte eólica, é dimensionada da seguinte maneira:

- Gerador Diesel - 1 x $10 \mathrm{~kW}$

- Gerador Fotovoltaico - 75 kW

- Baterias-67 x 1890Ah (7,55 kWh)

- Inversor - $20 \mathrm{~kW}$

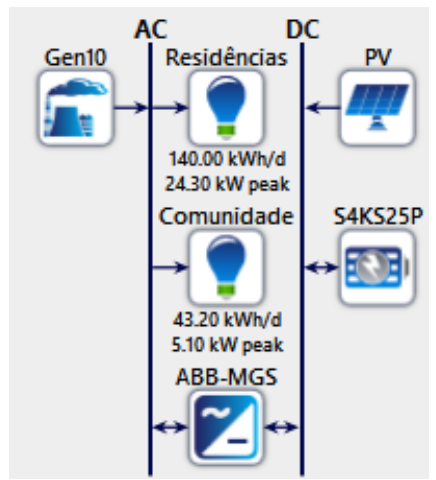

Figura 11 - Sistema Híbrido Solar - Diesel-Baterias Fonte: Software Homer Energy Pro

Os resultados apresentados na simulação desse sistema híbrido são destacados na Tabela 8, junto com a produção mensal na Figura 12.
Fonte: Software Homer Energy Pro

\begin{tabular}{c|c|c}
\hline Fonte & $\begin{array}{c}\text { Energia } \\
(\mathbf{k W h} / \mathbf{a n o})\end{array}$ & $\begin{array}{c}\text { Percentual } \\
\mathbf{( \% )}\end{array}$ \\
\hline Geração Fotovoltaica & $126.395,0$ & 98.7 \\
\hline Geração a Diesel & $1.707,0$ & 1.33 \\
\hline Total & $128.102,0$ & 100 \\
\hline Consumo & $66.830,0$ & - \\
\hline
\end{tabular}

GERAÇÃo FOTOVOLTAICA

GERAÇÃO A DIESEL

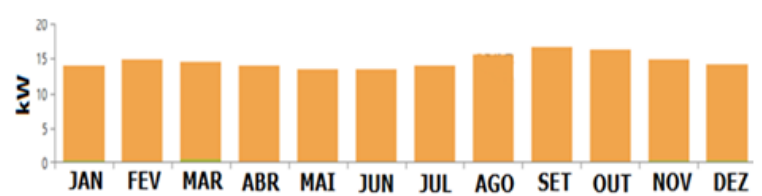

Figura 12 - Produção mensal de energia do sistema Fonte: Software Homer Energy Pro

No gráfico da Figura 13, é apresentado o comportamento do sistema para os primeiros dias do mês de Abril. A demanda representada em vermelho é atendida pelas fontes solar (cor laranja), baterias (cor verde) e em determinados momentos pelo gerador a diesel (cor preta). Neste período, observamos a alternância na geração entre as fontes solar e bateria, no qual a geração a diesel é somente ativada nos momentos em que as baterias não atendem integralmente a demanda solicitada do sistema.

Tabela 8 - Produção e Consumo anual de energia do sistema 
$\square$ GERAÇÃO FOTOVOLTAICA

GERAÇÃO A DIESEL

DEMANDA DO SISTEMA

SISTEMA DE ARMAZENAMENTO

Geração e Demanda (kW)

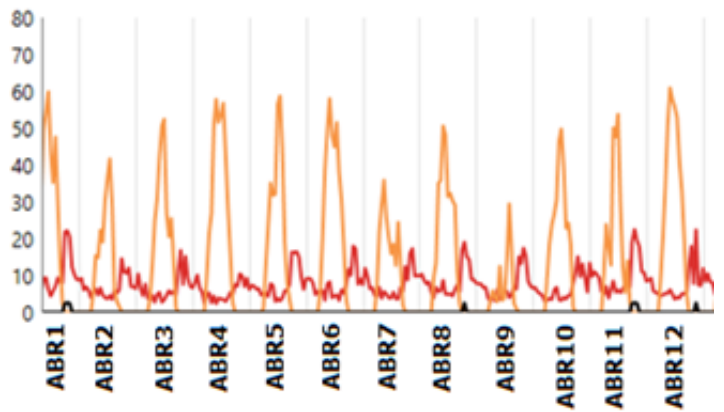

Descarga do Sistema de Armazenamento (kw)

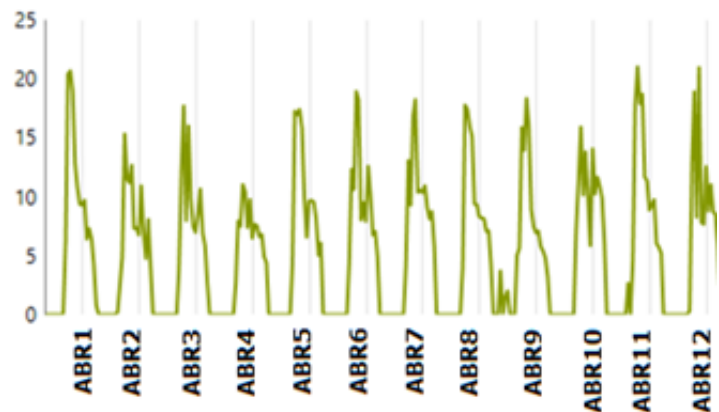

Figura 13 - Comportamento do sistema para os primeiros dias do mês de Abril

Fonte: Software Homer Energy Pro

\subsection{1 - Avaliação Financeira}

A análise dos custos estimado dos principais componentes do sistema é visto nas Tabelas 9 e 10.
Tabela 9 - Custos estimados associados ao sistema Solar-Eólico-Diesel-Baterias

\begin{tabular}{l|c|c|c}
\hline Componente & $\begin{array}{c}\text { Custo de } \\
\text { Investimento } \\
\text { inicial } \mathbf{( R \$ )}\end{array}$ & $\begin{array}{c}\text { Custo de } \\
\text { O\&M } \\
\text { (R\$/ano) }\end{array}$ & $\begin{array}{l}\text { Custo de } \\
\text { Reposição } \\
\text { (R\$/ano) }\end{array}$ \\
\hline Subsistema Solar & $375.112,14$ & $3.751,12$ & $-*$ \\
\hline $\begin{array}{l}\text { Subsistema de } \\
\text { Armazenamento }\end{array}$ & $402.000,00$ & $8.040,00$ & $12.034,08$ \\
\hline Gerador a Diesel & $25.000,00$ & $8.837,86$ & $-*$ \\
\hline Inversor & $35.000,00$ & 750,00 & 933,84 \\
\hline $\begin{array}{l}\text { Controle e } \\
\text { Supervisão }\end{array}$ & $40.000,00$ & $2.000,00$ & - \\
\hline Acessórios & $20.000,00$ & - & $-*$ \\
\hline $\begin{array}{l}\text { Rede de } \\
\text { Distribuição }\end{array}$ & $45.000,00$ & $2.500,00$ & $-*$ \\
\hline Total & $942.112,14$ & $25.878,98$ & $12.967,92$ \\
\hline
\end{tabular}

-* Não previstos custos de O\&M e reposição para os itens em destaque.

Tabela 10 - Indicadores de custos do sistema Fonte: Software Homer Energy Pro

\begin{tabular}{l|c}
\multicolumn{1}{c|}{ Indicador Financeiro } & Valor (R\$) \\
\hline Custo de Investimento Inicial (R\$) & $942.112,14$ \\
\hline Custo de O\&M - Vpa (R\$) & $202.972,46$ \\
\hline Custo de Reposição - Vpa (R\$) & $101.709,21$ \\
\hline Custo total (VPL) (R\$) & $1.246 .793,81$ \\
\hline Custo total anualizado (VPLA) (R\$) & $158.966,21$ \\
\hline
\end{tabular}

A energia consumida anual é igual a $66.830,0 \mathrm{Kwh} / a n o$ nessa configuração e de acordo a Tabela 10, temos um VPLA $=\mathrm{R} \$ 158.966,21$, logo o custo da energia é de $R \$ 2,37 / \mathrm{kWh}$.

\section{3 - Sistema Solar - Baterias}

A terceira configuração é um sistema contendo somente a fonte solar e baterias no fornecimento de energia. Na Tabela 11, temos os valores de produção e consumo registrados na simulação.

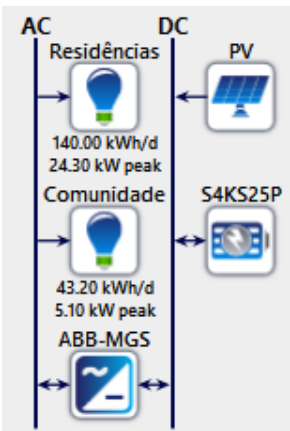

Figura 14 - Sistema Solar-Baterias Fonte: Software Homer Energy Pro 
O dimensionamento do sistema obtido na simulação para essa configuração é:

- Gerador Fotovoltaico - 87,1 kW

- Baterias-94 x 1890Ah (7,55 kWh)

- Inversor - $40 \mathrm{~kW}$

Tabela 11 - Produção e Consumo anual de energia do sistema

Fonte: Software Homer Energy Pro

\begin{tabular}{c|c|c}
\hline Fonte & $\begin{array}{c}\text { Energia } \\
\text { (kWh/ano) }\end{array}$ & $\begin{array}{c}\text { Percentual } \\
\text { (\%) }\end{array}$ \\
\hline Geração Fotovoltaica & $146.756,0$ & 100 \\
\hline Consumo & $66.837,0$ & - \\
\hline
\end{tabular}

Os valores de produção de energia mensal são indicados na Figura 15.

\section{GERAÇÃo FOTOVOLTAICA}

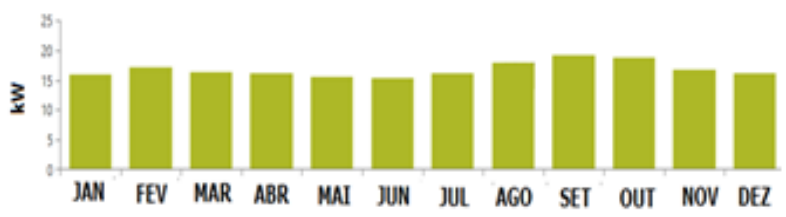

Figura 15 - Produção mensal de energia do sistema Fonte: Software Homer Energy Pro

No gráfico da Figura 16, observamos o comportamento da fonte solar (cor laranja) e a demanda do sistema (cor vermelha). Observa-se que a demanda é atendida somente em determinados períodos pela fonte solar e que a fontes solar gera um excesso de produção de energia nos momentos de atendimentos da demanda, logo a necessidade do acréscimo do banco de baterias (cor azul) para o armazenamento e suprimento de energia nos momentos de indisponibilidade da fonte solar, garantindo assim o atendimento e a confiabilidade do sistema.

\section{GERAÇÃO FOTOVOLTAICA \\ DEMANDA DO SISTEMA \\ SISTEMA DE ARMAZENAMENTO}
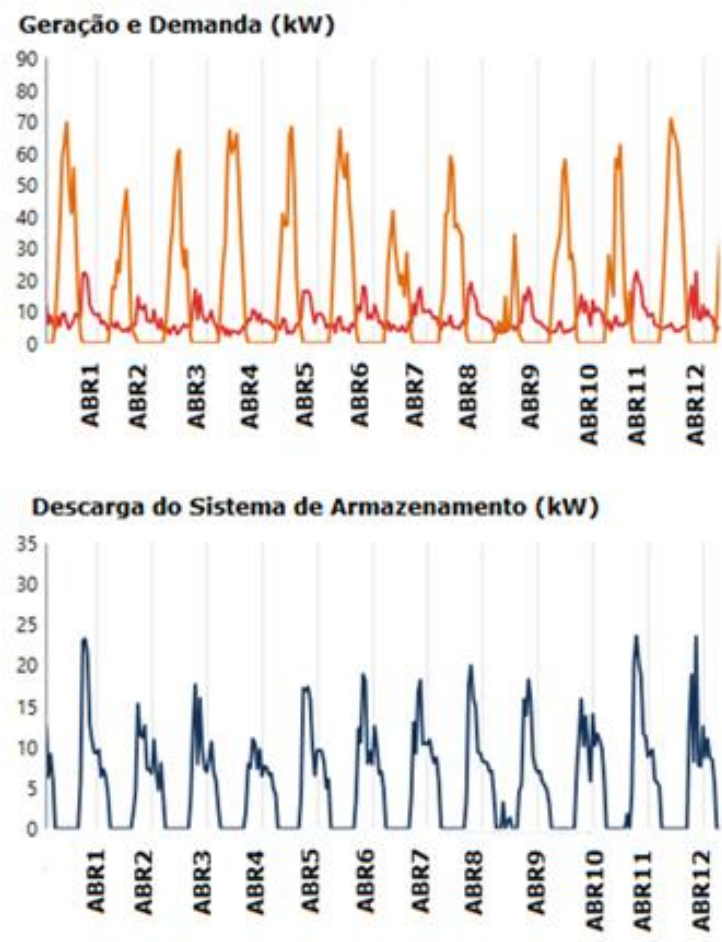

Figura 16 - Comportamento do sistema para os primeiros dias do mês de Abril Fonte: Software Homer Energy Pro

\subsection{1 - Avaliação Financeira}

A análise dos custos estimado dos principais componentes do sistema é visto nas Tabelas 12 e 13.

Tabela 12 - Custos estimados associados ao sistema Solar-Baterias

Fonte: Software Homer Energy Pro

\begin{tabular}{l|c|c|c}
\hline Componente & $\begin{array}{c}\text { Custo de } \\
\text { Investimento } \\
\text { inicial (R\$) }\end{array}$ & $\begin{array}{c}\text { Custo de } \\
\text { O\&M } \\
\text { (R\$/ano) }\end{array}$ & $\begin{array}{l}\text { Custo de } \\
\text { Reposição } \\
\text { (R\$/ano) }\end{array}$ \\
\hline Subsistema Solar & $435.540,19$ & $4.355,40$ & $-*$ \\
\hline $\begin{array}{l}\text { Subsistema de } \\
\text { Armazenamento }\end{array}$ & $564.000,00$ & $11.280,00$ & $10.623,40$ \\
\hline Inversor & $70.000,00$ & $1.500,00$ & $1.867,69$ \\
\hline $\begin{array}{l}\text { Controle e } \\
\text { Supervisão }\end{array}$ & $40.000,00$ & $2.000,00$ & - \\
\hline Acessórios & $20.000,00$ & - & $-*$ \\
\hline $\begin{array}{l}\text { Rede de } \\
\text { Distribuição }\end{array}$ & $45.000,00$ & $2.500,00$ & $-*$ \\
\hline Total & $1.129 .540,19$ & $21.635,40$ & $12.491,09$ \\
\hline
\end{tabular}

-* Não previstos custos de O\&M e reposição para os itens em destaque.

Tabela 13 - Indicadores de custos do sistema Fonte: Software Homer Energy Pro 


\begin{tabular}{l|c}
\multicolumn{1}{c|}{ Indicador Financeiro } & Valor (R\$) \\
\hline Custo de Investimento Inicial (R\$) & $1.129 .540,19$ \\
\hline Custo de O\&M - Vpa (R\$) & $169.689,47$ \\
\hline Custo de Reposição - Vpa (R\$) & $97.969,37$ \\
\hline Custo total (VPL) (R\$) & $1.397 .199,03$ \\
\hline Custo total anualizado (VPLA) (R\$) & $178.142,88$ \\
\hline
\end{tabular}

A energia consumida anual é igual a $66.837,0 \mathrm{kWh} /$ ano nessa configuração e de acordo a Tabela 13, temos um VPLA $=\mathrm{R} \$ 178.142,88$, logo o custo da energia é de $R \$ 2,66 / \mathrm{kWh}$.

Após a análise das simulações realizadas e a verificação dos três sistemas que apresentam melhor viabilidade financeira de implantação, observamos na Tabela 14 um comparativo dos sistemas avaliados anteriormente.

Tabela 14 - Comparativo do VPL e Custo da Energia dos Sistemas

\begin{tabular}{|c|c|c|}
\hline Sistema & VPL (R\$) & $\begin{array}{c}\text { Custo da } \\
\text { Energia } \\
\text { (R\$/kWh) }\end{array}$ \\
\hline $\begin{array}{l}\text { Sistema Solar - Eólico } \\
\text { - Diesel - Baterias }\end{array}$ & $1.357 .450,07$ & 2,59 \\
\hline $\begin{array}{l}\text { Sistema Solar - Diesel } \\
\text { - Baterias }\end{array}$ & $942.112,14$ & 2,37 \\
\hline $\begin{array}{l}\text { Sistema Solar - } \\
\text { Baterias }\end{array}$ & $1.129 .540,19$ & 2,66 \\
\hline
\end{tabular}

\section{Conclusões}

Ao analisarmos os resultados da simulação dos três sistemas podemos observar o seu comportamento no atendimento da demanda, onde logo após realizamos uma análise prévia econômica dos sistemas com base no VPL e no custo da energia do sistema.

De acordo com as simulações realizadas os três sistemas analisados foram os que apresentaram o menor custo de investimento e custo da energia. Porém, a configuração Solar Diesel - Baterias, apresentou-se como sendo a mais viável para uma aplicação por apresentar o menor VPL e custo da energia entre as três configurações analisadas.

Entretanto, o custo da energia apresentado pelo sistema Solar-Diesel-Baterias ainda é muito alto quando comparado ao custo da energia paga por consumidores que estão conectados ao sistema elétrico convencional.
Desta forma, os valores dos custos de energia dos sistemas híbridos avaliados estão distantes da realidade da comunidade isolada, sendo necessário o desenvolvimento de políticas públicas de acesso à energia por parte do governo de forma a fornecer subsídios para garantir que o sistema seja sustentável e as comunidades isoladas possam ter acesso à energia elétrica de qualidade.

\section{Referências}

[1] PINHO, J.T.; BARBOSA, C.F.O.; PEREIRA, E.J.S.; SOUZA, H.M.S.; BLASQUES, L.C.M.; GALHARDO, M.A.B.; MACEDO, W.N. Sistemas Híbridos: Soluções Energéticas para a Amazônia. $1^{a}$ edição. Ministério de Minas e Energia. Brasília, Brasil. Novembro, 2008

[2] ATLAS DE ENERGIA ELÉTRICA DO BRASIL/AGÊNCIA NACIONAL DE ENERGIA ELÉTRICA - 2ª Edição - Brasília:ANEEL,2005.

[3] ATLAS BRASILEIRO DE ENERGIA SOLAR / INSTITUTO NACIONAL DE PESQUISAS ESPACIAIS - INPE - 2a Edição - São José dos Campos, 2017.

[4] SISTEMAS ISOLADOS - PLANEJAMENTO DO ATENDIMENTO AOS SISTEMAS ISOLADOS/ EMPRESA DE PESQUISA ENERGÉTICA-EPE, 2016. 